\title{
CARENCIAS EN LOS SISTEMAS DE GESTIÓN DEL CONOCIMIENTO: UNA REVISIÓN BIBLIOGRÁFICA
}

\author{
Laura-Patricia Pinto-Prieto, Luis-Eduardo Becerra-Ardila y Luis-Carlos \\ Gómez-Flórez
}

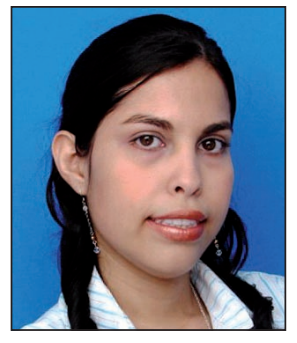

Laura-Patricia Pinto-Prieto es ingeniera de sistemas egresada con distinción cum laude de la Univ. Industrial de Santander (UIS), Colombia, en 2009. Es becaria del programa Jóvenes Investigadores del Instituto Colombiano para el Desarrollo de la Ciencia y la Tecnología Francisco José de Caldas (Colciencias). Estudiante de maestría en ingeniería industrial vinculada a los grupos de investigación STI (Sistemas y Tecnologías de Información) e Innotec (Centro para la gestión y la innovación tecnológica) de la UIS. Investiga en gestión del conocimiento, tecnologías de información, sistemas de información, e-learning, pensamiento sistémico y gestión tecnológica.

Universidad Industrial de Santander Facultad de Ingenierias Fisicomecánicas, Escuela de Ingeniería de Sistemas Grupo de Investigación en STI. Oficina LP 325 Carrera 27, Calle 9, Apartado aéreo 678 - Bucaramanga, Colombia lauris2687@hotmail.com

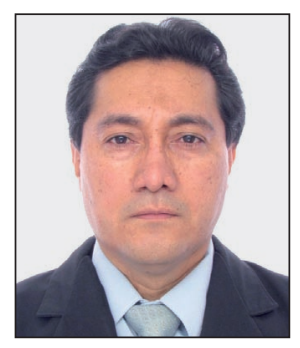

Luis-Eduardo Becerra-Ardila es ingeniero industrial egresado de la Universidad Industrial de Santander (UIS), Colombia, magister en administración del Instituto Tecnológico de Estudios Superiores de Monterrey y doctorando en ingeniería en la UIS. Es profesor asociado y jefe de división financiera de la misma universidad, y coordinador del proyecto SUMA (Towards Sustainable Financial Management of Universities in Latin America) financiado por la Comisión Europea, mediante el programa ALFA III; investigador del grupo de investigación Innotec (Centro para la Gestión y la Innovación Tecnológica); investiga en áreas de la gestión tecnológica y la administración de empresas.

Universidad Industrial de Santander Facultad de Ingenierias Fisicomecánicas, Escuela de Ingeniería de Sistemas Grupo de investigación Innotec Carrera 27, Calle 9, Apartado aéreo 678 - Bucaramanga, Colombia Ibecerra@uis.edu.co

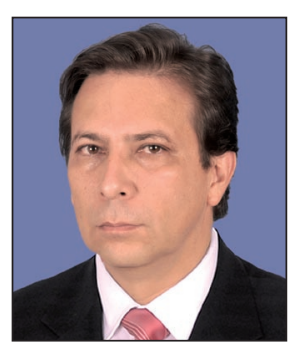

Luis-Carlos Gómez-Flórez es ingeniero de sistemas y magister en informática de la Universidad Industrial de Santander (Colombia). Profesor titular y director de investigacion y extensión de la Facultad de Ingenierías Fisicomecánicas de la misma universidad. Director del grupo de investigación STI (Sistemas y Tecnologías de la Información). Investigación sobre administración de la información y gestión del conocimiento en las organizaciones, auditoría y control en sistemas y tecnologías de información, gestión de procesos y calidad en ingeniería del software, pensamiento y metodología de los sistemas blandos, sistemas y tecnologías de la información en las organizaciones.

Universidad Industrial de Santander Facultad de Ingenierias Fisicomecánicas, Escuela de Ingeniería de Sistemas Grupo de Investigación en STI. Oficina LP 325 Carrera 27, Calle 9, Apartado aéreo 678 - Bucaramanga, Colombia Icgomezf@uis.edu.co

\section{Resumen}

La importancia del conocimiento como ventaja competitiva sostenible y el desarrollo de las tecnologías de información, han dado lugar a los sistemas de gestión del conocimiento (SGC), facilitando e impulsando los procesos de generación, codificación y transferencia del conocimiento en las organizaciones. El interés de profesionales, académicos e investigadores es creciente desde hace varios años, y la gestión de conocimiento es un tema en auge. Sin embargo, al examinar su estado en las organizaciones, se encuentran grandes dificultades en su incorporación. Mediante una revisión de la bibliografía se analizan los fallos usuales producidos en la implementación de los SGC. Los resultados reflejan la visión reduccionista que se centra sólo en las tecnologías de la información, revelándose la necesidad de una visión holística para poder tener éxito.

\section{Palabras clave}

Gestión del conocimiento, Conocimiento, Sistemas y tecnologías de información, Sistemas de gestión de conocimiento, Procesos de gestión de conocimiento, Fallos, Carencias. 
Title: Gaps in knowledge management systems: a bibliographical review

\begin{abstract}
The importance of knowledge as a sustainable competitive advantage and the increasing development of information technology (IT) have led to knowledge management systems (KMS), facilitating and encouraging the processes of generation, codification and transfer of knowledge in organizations. The interest of professionals, academics and researchers has been growing for several years, to the point that it can be stated that knowledge management is a topic on the rise. Nonetheless, if we review their status in organizations, there are great difficulties in their implementation, difficulties that cover various aspects. Through a review of the literature, this paper explores the present gaps in the implementation of KMS. The results reflect the dominance of a reductionist approach in this area, with a focus on IT, revealing the need for a holistic approach, guided by what makes sense in the organization.
\end{abstract}

\title{
Keywords
}

Knowledge management, Knowledge, Information systems and technologies, Knowledge management systems, Knowledge management processes, Gaps in knowledge management systems.

Pinto-Prieto, Laura-Patricia; Becerra-Ardila, Luis-Eduardo; Gómez-Flórez. "Carencias en los sistemas de gestión del conocimiento: una revisión bibliográfica”. El profesional de la información, 2012, mayo-junio, v. 21, n. 3, pp. 268-276.

http://dx.doi.org/10.3145/epi.2012.may.07

\section{Introducción}

Estamos en un momento de fuertes cambios económicos y sociales, dentro de la llamada sociedad del conocimiento, que con el avance de las tecnologías de la información (TI) permite a las organizaciones gestionar el conocimiento (GC) para apoyar procesos de innovación que generan nuevos productos y servicios, y por ende obtienen mejores ventajas competitivas.

Algunos autores (Tseng, 2008; Lindner; Wald, 2010; Kruger; Johnson, 2010) consideran las TI como facilitadoras de la GC, al hacer posible que el conocimiento fluya en la organización; sin embargo, revisada la bibliografía se hallaron varios gaps o lagunas para su correcta aplicación. Según Lin y Tseng (2005) citado por Li y Chang, (2009), la mayoría de fallos se presentan entre la planificación y la implementación de proyectos de sistemas de GC, y en la diferencia entre los conocimientos necesarios según la percepción de los directivos y la de los demás empleados.

En este artículo se destaca la importancia de tratar la situación desde una visión holística o sistémica, específicamente desde el paradigma perspectivista de Checkland (1993), permitiendo identificar los conocimientos relevantes y la tecnología apropiada para la GC en la organización y de esta manera contribuir a reducir los gaps mencionados.

Sin las TI sería imposible realizar los cambios necesarios en las organizaciones para implementar la gestión del conocimiento

\section{Gestión del conocimiento y tecnologías de información}

A lo largo del tiempo han existido diversos enfoques que explican la obtención de ventajas competitivas de una orga- nización. La evolución de dichos enfoques ha dado lugar a la Visión basada en las competencias, que se ha convertido en uno de los paradigmas dominantes en la dirección estratégica (Camisón, 2002; Foss, 1998).

Dentro de esta visión se encuentran los enfoques basados en los recursos, capacidades dinámicas y conocimiento.

En relación con el conocimiento, varios autores lo consideran la fuente de ventaja competitiva sostenible de la organización: Nonaka (1994), Nonaka y Takeuchi (1995), Spender (1996), Grant (1996), Kogut y Zander (1992), Garud (1997), (Forés, 2010; Alavi; Leidner, 2001) especialmente importante por su carácter intangible e inimitable (Prahalad; Hamel, 1990; Grant, 1991; Stalk; Evans; Shulman, 1992; Knudsen, 1996; Teece; Pisano; Shuen, 1997).

Alavi y Leidner (2001) mencionan que dependiendo de la perspectiva bajo la cual se vea el conocimiento (transformación de datos a información, estado de la mente, objetos, procesos, facilidad de acceso a la información, capacidad) la GC y las TI cumplen un rol determinado. Si el conocimiento es visto como un proceso de aplicación de la experiencia (Carlsson et al., 1996; McQueen, 1998; Zack, 1998), o como un objeto que puede ser almacenado y manipulado (Zack, 1998), la GC es un proceso que comprende crear, aplicar y transferir conocimientos en la empresa, para obtener un beneficio (Macintosh, 1996; Wiig, 1997; Andreu; Sieber, 1999; Alavi; Leidner, 2001).

En cuanto a las actividades y/o procesos que componen la GC no se ha llegado a un consenso. Muchos autores han creado modelos para explicar cuáles son, entre ellos Forés (2010), quien cita los modelos:

- crecimiento de conocimiento (Kogut; Zander, 1992);

- espiral de creación de conocimiento (Nonaka; Takeuchi, 1995);

- transferencia y transformación de conocimiento (Hedlund; Nonaka, 1993; Hedlund, 1994);

- aprendizaje (Leonard-Barton, 1995); 
- las 4-i del aprendizaje organizativo (Crossan; Lane; White, 1999);

- cinco ciclos de aprendizaje (Sánchez, 2001);

- ciclo evolutivo del conocimiento (Zollo; Winter, 2002).

Analizando a varios autores, Jaime, Gardoni y Frank (2005) listan las siguientes actividades de GC: identificación, adquisición, selección, estructuración, creación, transferencia, uso, codificación y medición. Por su lado, Alavi y Leidner (2001) consideran cuatro procesos de GC: creación del conocimiento, almacenamiento, transferencia y aplicación.

Las TI son un facilitador de la GC y permiten una mejor comunicación y flujo del conocimiento en la organización. Algunos autores como Khandelwal y Gottschalk (2003), Spiegler (2003) y Sher y Lee (2004), destacan la importancia de la aplicación de las TI para dar soporte en los procesos de creación y transferencia de conocimiento. Schindler y Eppler (2003); Karlsen y Gottschalk (2004); Adenfelt y Lagerström (2006); Hanisch et al. (2009); y Lindner y Wald, (2010) indican que las TI se encuentran entre los factores de éxito más relevantes en la implementación de proyectos de carácter temporal de GC en organizaciones.

Dos conceptos ampliamente utilizados para relacionar las $\mathrm{TI}$ con la GC son los sistemas basados en el conocimiento (SBC) y los sistemas de gestión de conocimiento (SGC).

- Los primeros pretenden entender e imitar los conocimientos humanos haciendo uso de técnicas de inteligencia artificial (Wiig, 1997; Hendriks; Vriens, 1999; Huang, 2009; Chang, 2011). Sus principales componentes son: una base de conocimiento, un motor de inferencia, una herramienta de ingeniería de conocimiento y una interfaz de usuario (Wiig, 1997; Hendriks; Vriens, 1999).

- Los SGC son softwares diseñados para soportar los procesos de GC, y son los que principalmente nos referimos aquí (Von-Krogh, 1998; Baroni-de-Carvalho; Tavares, 2001; Croasdell et al., 2003; Benbya; Passiante; Aissa, 2004; Nevo; Chan, 2007; Richardson; Courtney; Haynes, 2006).

Sin embargo, pese a las bondades de las TI para la GC, se han identificado algunos gaps que dificultan la obtención de resultados satisfactorios en la implementación y uso de los SGC en las organizaciones.

\section{Fallos en los SGC. Necesidad de una visión holística}

Diversos estudios analizan las causas del fracaso de los proyectos de GC, sus limitaciones, carencias y enfoque dominante. Por ejemplo Alavi y Leidner (2001) indican que mientras la mayor parte de los estudios teóricos sobre la GC se centra en la estrategia de la organización, existen pocas investigaciones empíricas sobre el apoyo de las TI a la GC.

Varios investigadores se han referido al excesivo énfasis puesto en las tecnologías. Según Nonaka y Takeuchi (1995), Anand, Manz y Glick (1998),
Davenport y Prusak (1998), referenciados por Chua (2004), el éxito de la aplicación de la GC no se puede basar en el despliegue de una solución tecnológica por sí sola. Las investigaciones han revelado que la mayor dificultad en la GC es cambiar el comportamiento de la gente, y el mayor impedimento para la transferencia de conocimiento actual es la cultura organizativa. Superar las limitaciones tecnológicas, por el contrario, suele ser un asunto trivial (Ruggles, 1998). La tecnología existente no puede ser un sustituto completo del contacto cara a cara, que es crucial para la construcción de una cultura de intercambio de conocimientos (Roberts, 2000). Tampoco puede sustituir la interacción social humana, la interactividad entre los individuos necesarios para la creación de conocimiento (Fahey; Prusak, 1998).

El énfasis sobre los SGC se debe a que prevalece una visión reduccionista, centrada en las TI, en lugar de una visión holística. Esta situación también está presente en la elaboración de sistemas de información y en el uso de TI para la mejora de procesos organizativos (Davenport; Prusak, 1998; Barceló; Baglietto, 2001; Olave; Gómez, 2002; 2007).

Chua (2004) afirma que "la tecnología es capaz de superar las barreras del tiempo y el espacio que de otra manera serían limitantes en las actividades de gestión. También sirve como repositorio en el que el conocimiento puede ser contrastado, almacenado y recuperado de manera eficiente". Este autor se centra en cómo reducir la distancia entre los consultores y los tecnólogos, y plantea un modelo de arquitectura para plasmar cómo pueden ser usadas las TI para soportar los procesos de GC. En la arquitectura identifica tres partes:

- infraestructura (almacenamiento y comunicación);

- conocimiento (creación, intercambio y reutilización); y

- presentación (personalización y visualización).

Lin y Tseng (2005) identificaron cinco gaps o carencias en la GC e ilustran la relación entre las actividades de gestión y el desempeño corporativo. A partir de esto, Tseng (2008) propone un marco conceptual que explora el rol y los efectos de las TI en los SGC, y cómo mejorar la eficacia y la eficiencia. La figura 1 presenta esos 5 gaps y cómo las TI pueden ayudar a reducirlos.

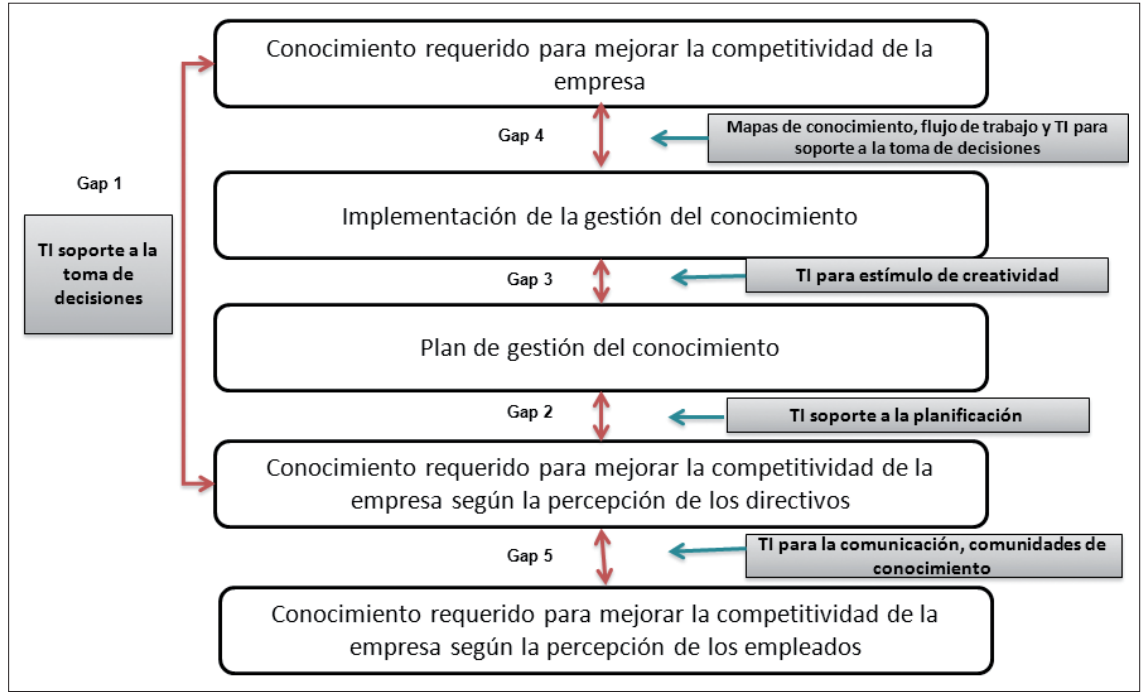

Figura 1. Gaps o fallos en la gestión del conocimiento e influencia de las TI 
Los gaps se encuentran entre los conocimientos requeridos por la organización según la percepción de los directivos, y según la de los demás empleados. Existen inconsistencias entre la planificación e implementación de los SGC, que no son diseñados de tal manera que estén alineados con los objetivos y estrategias de la organización, y por lo tanto los conocimientos gestionados no son los requeridos para lograr los objetivos. La tabla 1 presenta los principales resultados obtenidos por Tseng (2008) al analizar el efecto de las TI sobre los gaps mencionados anteriormente.

Las TI para la GC pueden contribuir a la reducción de las lagunas y carencias, pero para ello cuando se compra o elabora un software para GC se tiene que tener en cuenta el dominio de la aplicación, el tipo de conocimiento y los procesos que van a ser soportados. En la tabla 2, tomada de Rodríguez et al. (2008), se listan varios autores y el enfoque de sus artículos relacionados con las TI para la GC. Sin embargo, todavía son pocos los estudios en los que se analiza la organización sistémicamente, teniendo en cuenta sus objetivos y estrategias. En el desarrollo de los SGC prevalece una visión excesivamente simplista.

Como se puede ver en la tabla 2, la mayoría de los trabajos se han centrado en analizar cómo las TI soportan las actividades de GC, siendo vistas como simples herramientas. Con este panorama si una organización desea diseñar un SGC es muy probable que se fije en las tecnologías, dejando a un lado otros factores mucho más relevantes que contribuirían a determinar si realmente es factible la GC bajo las condiciones actuales de la organización.

Por desgracia la definición de las tecnologías por parte de los técnicos y de la estrategia de GC por parte de la alta dirección se realizan independientemente, sin tener una visión holística del problema, y cada uno piensa que el SGC ya se adaptará. Esto se constata con los planteamientos de

\begin{tabular}{|c|c|}
\hline Gap & Influencia de las TI \\
\hline Gap 1 & $\begin{array}{l}\text { - El principal beneficio de las TI es facilitar a los directivos la } \\
\text { identificación de un conocimiento valioso. } \\
\text { - Las TI son sólo un medio para fomentar el conocimiento. }\end{array}$ \\
\hline Gap 2 & $\begin{array}{l}\text { - Los repositorios de GC facilitan la captura, difusión y al- } \\
\text { macenamiento de conocimiento. } \\
\text { - La necesidad de cambios incesantes en el conocimiento y } \\
\text { el sistema estimula la creación de conocimiento y la inno- } \\
\text { vación. } \\
\text { - Los repositorios de conocimiento ayudan a los gerentes a } \\
\text { planificar la GC, intensificar el aprendizaje organizativo, y } \\
\text { mejorar la planificación y toma de decisiones. }\end{array}$ \\
\hline Gap 3 & $\begin{array}{l}\text { - Los SGC estimulan la creatividad y la innovación en la or- } \\
\text { ganización post-industrial y permiten monitorear y con- } \\
\text { trolar la ejecución de los planes de GC. }\end{array}$ \\
\hline Gap 4 & $\begin{array}{l}\text { - Es necesario un sistema de medición completa que per- } \\
\text { mita a la organización evaluar si la empresa mejora su } \\
\text { competitividad después de la implementación del SGC. } \\
\text { Esto se puede realizar con herramientas como el Balanced } \\
\text { scorecard y los mapas estratégicos. }\end{array}$ \\
\hline Gap 5 & $\begin{array}{l}\text { - Software de trabajo en grupo, de soporte de decisiones } \\
\text { grupales, de flujo de trabajo (work flow), videoconferen- } \\
\text { cias e intranets pueden facilitar el intercambio de infor- } \\
\text { mación interna, la discusión y la comunicación en las or- } \\
\text { ganizaciones. }\end{array}$ \\
\hline
\end{tabular}

Tabla 1. Uso de las TI y posibles fallos o gaps de los SGC (basado en Tseng, 2008)
Rubenstein et al. (2001), quienes realizaron un análisis de marcos de trabajo (frameworks) para la GC. Los clasifican en:

- descriptivos (proporcionan orientación sobre los tipos de procedimientos de GC, sin dar detalles específicos de cómo pueden ó deben llevarse a cabo), y

- prescriptivos (identifican atributos de la GC importantes por su influencia en el éxito o el fracaso de las iniciativas).

Los anteriores autores mencionan que en la literatura la mayor parte de los marcos de trabajo son prescriptivos y por lo tanto están más orientados hacia tareas.

Parte de las causas de los gap en los SGC, está en la forma en cómo se han visto los SGC, en la mayoría de los casos como: "las herramientas, tecnologías o software diseñados para soportar los procesos de generación, codificación y transferencia de conocimiento.

\section{Metodología de sistemas blandos: una alternativa para reducir la distancia entre gestores y técnicos}

Para entender por qué es necesario ver la GC desde un enfoque sistémico es necesario tener claro qué es el pensamiento sistémico, dentro del cual existen diversos enfoques o perspectivas, que Jackson describió en 2003. En lo relacionado con la GC, la perspectiva que más sobresale es la llamada metodología de sistemas suaves o blandos (MSB) -soft systems methodology-, que busca definir la transformación de una organización mediante el debate y el acuerdo entre los actores involucrados. es un enfoque sistémico para abordar situaciones problemáticas. Proporciona un marco conceptual para personas que deben enfrentarse con situaciones problemáticas y no tienen una definición formal del problema.

La MSB proporciona herramientas y principios que ayudan a identificar los conocimientos relevantes y necesarios y la forma de gestionarlos. De esta forma los criterios no son impuestos desde la alta dirección sino que son definidos en conjunto con todos los empleados, garantizando que la implementación esté acorde con la planificación, dado que todos los actores han aceptado sus acuerdos y desacuerdos.

Otros autores (Kingston; Macintosh, 2000; Rubenstein et al., 2001; Skok; Kalmanovitch, 2005; Butler et al., 2008; Kotiadis; Robinson, 2008; Georgiou, 2008) destacan así mismo la utilidad del método de sistemas blandos. Los trabajos de Yim et al. (2004), Georgiou (2008), Powell y Swart (2010) aplican el pensamiento sistémico a los procesos de toma de decisiones.

Como se ha visto, existen muchas oportunidades de investigación en torno a los SGC, principalmente cuando se 


\begin{tabular}{|c|c|c|c|}
\hline Autor & Año & Propósito & Aspectos cubiertos \\
\hline $\begin{array}{l}\text { Borghoff y } \\
\text { Pareschi }\end{array}$ & 1997 & Clasificar las tecnologías para la GC & $\begin{array}{l}\text { Actividades de GC } \\
\text { Estructura del conocimiento (tácito o explícito) }\end{array}$ \\
\hline $\begin{array}{l}\text { Newman y } \\
\text { Conrad }\end{array}$ & 2000 & Organizar y clasificar los métodos, practicas y tecnologías de la GC & $\begin{array}{l}\text { Actividades de GC } \\
\text { Estructura del conocimiento (tácito o explícito) } \\
\text { Dominio de aplicación. }\end{array}$ \\
\hline Alavi y Leidner & 2001 & Analizar el rol de los SGC en la GC organizativa & Actividades de GC \\
\hline Binney & 2001 & Clasificar aplicaciones mencionadas en la literatura & $\begin{array}{l}\text { Uso del conocimiento } \\
\text { Actividades de GC }\end{array}$ \\
\hline Marwick & 2001 & Describir características de las tecnologías de GC & $\begin{array}{l}\text { Actividades de GC } \\
\text { Estructura del conocimiento (tácito o explícito) }\end{array}$ \\
\hline Rus et al. & 2001 & $\begin{array}{l}\text { Clasificar las tecnologías para la GC usadas en ingeniería del } \\
\text { software }\end{array}$ & $\begin{array}{l}\text { Uso del conocimiento } \\
\text { Dominio del conocimiento }\end{array}$ \\
\hline $\begin{array}{l}\text { Tiwana y } \\
\text { Ramesh }\end{array}$ & 2001 & Clasificar las tecnologías de GC y describir algunos SGC & $\begin{array}{l}\text { Dominio de aplicación (utilización de los conocimien- } \\
\text { tos) } \\
\text { Actividades de GC } \\
\text { Cuestiones técnicas (específicos para sistemas basa- } \\
\text { dos en la web) }\end{array}$ \\
\hline $\begin{array}{l}\text { Valente y } \\
\text { Housel }\end{array}$ & 2002 & Analizar y comparar herramientas de GC & $\begin{array}{l}\text { Actividades de GC } \\
\text { Estructura de conocimiento } \\
\text { Dominio de aplicación }\end{array}$ \\
\hline $\begin{array}{l}\text { Woitsch y } \\
\text { Karagiannis }\end{array}$ & 2002 & Definir funcionalidades requeridas de un SGC empresarial & $\begin{array}{l}\text { Actividades de GC } \\
\text { Estructura de conocimiento } \\
\text { Cuestiones técnicas }\end{array}$ \\
\hline $\begin{array}{l}\text { Kankanhalli } \\
\text { et al. }\end{array}$ & 2003 & Analizar el rol de las TI en la GC en un grupo de organizaciones & Uso del conocimiento \\
\hline $\begin{array}{l}\text { Van-Elst } \\
\text { et al. }\end{array}$ & 2003 & Clasificar y analizar enfoques de GC basados en agentes & $\begin{array}{l}\text { Actividades de GC } \\
\text { Dominio de la aplicación }\end{array}$ \\
\hline $\begin{array}{l}\text { Qureshi } \\
\text { et al. }\end{array}$ & 2004 & Analizar herramientas de groupware como tecnologías de GC & $\begin{array}{l}\text { Actividades de GC } \\
\text { Dominio de aplicación (utilización de los conocimien- } \\
\text { tos) } \\
\text { Cuestiones técnicas (relacionadas con groupware } \\
\text { herramientas) }\end{array}$ \\
\hline Chua & 2004 & $\begin{array}{l}\text { Desarrollar una arquitectura de sistemas de GC que busca reducir la } \\
\text { brecha entre los consultores y tecnólogos. }\end{array}$ & $\begin{array}{l}\text { Actividades de GC } \\
\text { Cuestiones técnicas }\end{array}$ \\
\hline Banerjee & 2005 & $\begin{array}{l}\text { Analizar cómo las herramientas de GC son usadas en la organiza- } \\
\text { ción }\end{array}$ & $\begin{array}{l}\text { Actividades de GC } \\
\text { Dominio de aplicación (uso de conocimiento) } \\
\text { Cuestiones técnicas (distribución de fuentes de cono- } \\
\text { cimiento, temporalidad del acceso al conocimiento) }\end{array}$ \\
\hline Rao & 2005 & Clasifica y describe características de las tecnologías de GC & $\begin{array}{l}\text { Actividades de GC } \\
\text { Dominio de aplicación (uso de conocimiento) } \\
\text { Cuestiones técnicas (características de conocimientos } \\
\text { y de tipos específicos de herramientas) }\end{array}$ \\
\hline $\begin{array}{l}\text { Vizcaíno } \\
\text { et al. }\end{array}$ & 2005 & Evaluar si los sistemas colaborativos soportan las actividades de GC & Actividades de GC \\
\hline $\begin{array}{l}\text { Rodríguez } \\
\text { et al. }\end{array}$ & 2008 & $\begin{array}{l}\text { Presenta un marco que puede utilizarse para analizar los sistemas } \\
\text { de información como facilitadores del flujo de conocimientos en } \\
\text { los procesos de la organización }\end{array}$ & $\begin{array}{l}\text { Dominio de la aplicación } \\
\text { Actividades de la GC } \\
\text { Estructura de conocimiento } \\
\text { Cuestiones técnicas }\end{array}$ \\
\hline $\begin{array}{l}\text { Gottschalk } \\
\text { y Dean }\end{array}$ & 2010 & $\begin{array}{l}\text { Crear un modelo de escenario para los sistemas de gestión del } \\
\text { conocimiento en la vigilancia de la delincuencia financiera }\end{array}$ & $\begin{array}{l}\text { Dominio de la aplicación } \\
\text { Cuestiones técnicas }\end{array}$ \\
\hline Zhao & 2010 & $\begin{array}{l}\text { Desarrollar un marco de trabajo para facilitar el aprendizaje de los } \\
\text { docentes }\end{array}$ & $\begin{array}{l}\text { Dominio de la aplicación } \\
\text { Actividades de GC } \\
\text { Cuestiones técnicas }\end{array}$ \\
\hline
\end{tabular}

Tabla 2. Autores y enfoque de sus artículos sobre técnicas para SGC (adaptado de Rodríguez et al., 2008)

busca relacionar el pensamiento sistémico con la GC. Pese a los intentos realizados por reducir los gaps mencionados no se ha conseguido un gran avance, dado que todavía prevalece la posición mecanocéntrica (centrada en la máquina) en lugar de la centrada en el ser humano (Olave; Gómez, 2007).

\section{Conclusiones}

La GC se incorpora cada vez más a las organizaciones para mejorar su rendimiento, pero existen carencias que dificultan su completa implementación de acuerdo con los objetivos y estrategias de la organización, y se queda sólo en gestión de la información. Esto se debe a un enfoque centrado 
únicamente en los aspectos técnicos, dejando a un lado factores necesarios para la GC como la cultura organizativa, las políticas y objetivos de la organización y sobre todo el capital humano; en otras palabras, dejando de lado el verdadero sistema.

Una de las posibles causas de este problema radica en la forma reduccionista cómo se presentan los SGC, como si la simple tecnología fuera suficiente para implementar un sistema de gestión del conocimiento.

\section{Bibliografía}

Adenfelt, Maria; Lagerström, Katarina. "Enabling knowledge creation and sharing in transnational projects". Intl journal of project management, 2006, v. 24, n. 3, pp. 191198.

http://dx.doi.org/10.1016/j.ijproman.2005.09.003

Alavi, Maryam; Leidner, Dorothy. "Review: knowledge management and knowledge management systems: conceptual foundations and research issues". MIS quarterly, 2001, v. 25, n. 1, pp. 107-136.

http://dx.doi.org/10.2307/3250961

Anand, Vikas; Manz, Charles; Glick, William. "An organizational memory approach to information management". Academy of management review, 1998, v. 23, n. 4, pp. 796 800.

http://dx.doi.org/10.5465/AMR.1998.1255639

Andreu, Rafael; Sieber, Sandra. "La gestión integral del conocimiento y del aprendizaje". Economía industrial, 1999, v. 326, pp. 63-72.

Banerjee, Ritendra. "A fool with a tool is still a fool". En: Rao, Madanmohan (ed.). Knowledge management tools and techniques. Amsterdam: Elsevier, 2005, pp. 283-292.

Barceló, María; Baglietto, Alicia. Hacia una economía del conocimiento. Price Waterhouse Coopers, ESIC Editorial, 2001. ISBN: 8473562720

Baroni-de-Carvalho, Rodrigo; Tavares, Marta. "Using information technology to support knowledge conversion processes". Information research, 2001, v. 7, n. 1.

http://InformationR.net/ir/7-1/paper118.html

Benbya, Hind; Passiante, Giuseppin; Aissa, Nassi. “Corporate portal: a tool for knowledge management synchronization". Intl journal of information management, 2004, June, v. 24, n. 3, pp. 201-220.

http://choo.fis.utoronto.ca/fis/courses/lis2102/Readings/ benbya.pdf

http://dx.doi.org/10.1016/j.ijinfomgt.2003.12.012

Binney, Derek. "The knowledge management spectrum - understanding the KM landscape". Journal of knowledge management, 2001, v. 5, pp. 33-42.

http://dx.doi.org/10.1108/13673270110384383

Borghoff, Uwe; Pareschi, Remo. "Information technology for knowledge management". Journal of universal computer science, 1997, v. 3, n. 8, pp. 835-842.

http://www.jucs.org/jucs_3_8/information_technology_ for_knowledge/Borghoff_U_M.pdf
Butler, Tom; Feller, Joseph; Pope, Andrew; Emerson, Bill; Murphy, Ciaran. "Designing a core IT artefact for knowledge management systems using participatory action research in a government and a non-government organisation". Journal of strategic information systems, 2008, v. 17, n. 4, pp. 249267.

http://dx.doi.org/10.1016/j.jsis.2007.10.002

Carlsson, Sven; ElSawy, Omar; Eriksson, Innger; Raven, Arjan. "Gaining competitive advantage through shared knowledge creation: in search of a new design theory for strategic information systems". En: Procs of the $4^{\text {th }}$ European conf on information systems, Lisbon, 1996.

Camisón, César. "Las competencias distintivas basadas en activos intangibles". En: Morcillo, Patricio; Fernández-Aguado, Javier. Nuevas claves para la dirección estratégica. Barcelona: Ariel, 2002, pp. 117-151. ISBN: 8434421917

Chang, Wei-Lun. "iValue: a knowledge-based system for estimating customer prospect value". Knowledge-based systems, 2011, v. 24, n. 8, pp. 1181-1186.

http://dx.doi.org/10.1016/j.knosys.2011.05.004

Checkland, Peter. Pensamiento de sistemas, práctica de sistemas. México: Limusa Noriega, 1993. ISBN: 978 9681845254

Chua, Alton. "Knowledge management system architecture: a bridge between KM consultants and technologists". Int/ journal of information management, 2004, v. 24, n. 1, pp. 87-98.

http://dx.doi.org/10.1016/j.ijinfomgt.2003.10.003

Croasdell, David; Jennex, Murray; Zhihong, Yu; Christianson, Tony; Chakradeo, Meenal; Makdum, Wagas. "A meta-analysis of methodologies for research in knowledge management, organizational learning and organizational memory: five years at HICSS". System Sciences. Procs of the $36^{\text {th }}$ Hawaii intl conf on system sciences, 2003.

http://www.computer.org/csdl/proceedings/hicss/2003/ 1874/04/187440110a-abs.html

http://doi.ieeecomputersociety.org/10.1109/HICSS. 2003.1174253

Crossan, Mary; Lane, Henry; White, Roderick. "An organizational learning framework: from intuition to institution". Academy of management review, 1999, v. 24, n. 3, pp. 522537.

Davenport, Tom; Prusak, Laurence. Working knowledge: how organizations manage what they know. Boston: Harvard Business School Press, 1998.

Fahey, Liam; Prusak, Laurence. "The eleven deadliest sins of knowledge management". California management review, 1998, v. 40, n. 3, pp. 265-276.

http://www.comp.dit.ie/dgordon/Courses/Research Methods/Countdown/11DeadliestSins.pdf

Forés, Beatriz. Prácticas organizativas, capacidades dinámicas y desempeño económico. Tesis doctoral. Universitat Jaume I, 2010.

Foss, Nicolai. "Introduction: the emerging competence perspective". En: Foss, Nicolai J.; Knudsen, Christian (eds.). To- 
wards a competence theory of the firm. Londres: Routledge, 1998, pp. 1-12. ISBN: 9780415144728

Garud, Raghu. "On the distinction between know-how, know-why and know-what in technological systems". Advances in strategic management, 1997, v. 14, pp. 81-101. http://www.personal.psu.edu/users/r/u/rug14/48.On_the_ Distinction_between_know-how_kno.pdf

Georgiou, Ion. "Making decisions in the absence of clear facts". European journal of operational research, 2008, v. 185, pp. 299-321.

http://dx.doi.org/10.1016/j.ejor.2006.12.038

Gottschalk, Petter; Dean, Geoff. "Stages of knowledge management systems in policing financial crime". Int journal of law, crime and justice, 2010, v. 38, n. 3, pp. 94-108. http://dx.doi.org/10.1016/j.ijlcj.2010.09.001

Grant, Robert. "The resource-based theory of competitive advantages: implications for strategy formulation". California management review, 1991, v. 33, n. 3, pp. 114-135. http://www.skynet.ie/ karen/Articles/Grant1_NB.pdf

Grant, Robert. "Toward a knowledge-based theory of the firm". Strategic management journal, 1996, v. 17, n. 7, pp. 109-122.

http://www.wedb.net/download/valoracao/aula_7/grant_ 1996.pdf

Hanisch, Bastian; Müller, Ana; Lindner, Frank; Wald, Andreas. "Knowledge management in temporary project environments". Journal of knowledge management, 2009, v. 13, n. 4, pp. 148-160.

http://dx.doi.org/10.1108/13673270910971897

Hedlund, Gunnar. "A model of knowledge management and the n-form corporation". Strategic management journal, 1994, v. 15, pp. 73-90.

http://dx.doi.org/10.1002/smj.4250151006

Hedlund, Gunnar; Nonaka, Ikujiro. "Models of knowledge management in the West and Japan". En: Lorage, Peter; Chakravarthy, Bala; Roos, Johan. Implementing strategic processes. Blackwell, 1993, pp. 117-144. ISBN: 978 0631185659

Hendriks, Paul; Vriens, Dirk. "Knowledge-based systems and knowledge management: friends or foes?" Information \& management, 1999, v. 35, n. 2, pp. 113-125. http://dx.doi.org/10.1016/S0378-7206(98)00080-9

Huang, Hao-Chen. "Designing a knowledge-based system for strategic planning: a balanced scorecard perspective". Expert systems with applications, 2009, v. 36, n. 1, pp. 209-218. http://dx.doi.org/10.1016/j.eswa.2007.09.046

Jaime, Astrid; Gardoni, Mickael; Frank, Christian. "Communications tools in research projects to support semi and non structured information". Journal of systemics, cybernetics and informatics, 2005, v. 3, n. 3, pp. 85-93.

http://www.iiisci.org/journal/CV\$\$sci/pdfs/P250685.pdf

Jackson, Michael. Systems thinking: creative holism for managers. John Wiley, 2003. ISBN: 9780470845226

Kankanhalli, Atreyi; Tanudidjaja, Fransiska; Sutanto, Juli- ana; Tan, Bernard. "The role of IT in successful knowledge management initiatives". Communications of the ACM, 2003, v. 46, n. 9, pp. 69-73.

http://dx.doi.org/10.1145/903893.903896

Karlsen, Jan; Gottschalk, Petter. "Factors affecting knowledge transfer in IT projects". Engineering management journal, 2004, v. 16, n. 1, pp. 3-10.

Khandelwal, Vijay; Gottschalk, Petter. "Information technology support for interorganizational knowledge transfer: an empirical study of law firms in Norway and Australia". Information resources management journal, 2003, v. 16, n. 1, pp. 14-23.

http://dx.doi.org/10.4018/irmj.2003010102

Kingston, John; Macintosh, Ann. "Knowledge management through multi-perspective modelling: representing and distributing organizational memory". Knowledge-based syste$m s, 2000$, v. 13, n. 2-3, pp. 121-131.

http://dx.doi.org/10.1016/S0950-7051(00)00053-8

Knudsen, Christian. "The competence perspective: a historical view". En: Foss, Nicolai J.; Knudsen, Christian (eds.). Towards a competence theory of the firm, 1996, pp. 13-37. ISBN: 9780415144728

Kogut, Bruce; Zander, Udo. "Knowledge of the firm, combinative capabilities, and the replication of technology". Organization science, 1992, v. 3, n. 3, pp. 383-397.

http://zonecours.hec.ca/documents/H2008-1-1548530. seance10knowledgeofthefirm.pdf

Kotiadis, Kathy; Robinson, Stewart. "Conceptual modelling: knowledge acquisition and model abstraction". Procs of the 2008 Winter simulation, 2008, pp. 951-958.

http://www.informs-sim.org/wsc08papers/114.pdf

Kruger, Neels; Johnson, Roy. "Information management as an enabler of knowledge management maturity: A South African perspective". Intl journal of information management, 2010, v. 30, pp. 57-67.

http://137.215.9.22/bitstream/handle/2263/11613/Kru ger_Information(2009).pdf?sequence $=1$

http://dx.doi.org/10.1016/j.ijinfomgt.2009.06.007

Leonard-Barton, Dorothy. Wellsprings of knowledge: Building and sustaining the sources of innovation. Boston: Harvard Business School Press, 1995. ISBN: 9780875848594

Li, Sheng-Tun; Chang, Won-Chen. "Exploiting and transferring presentational knowledge assets in R\&D organizations". Expert systems with applications, 2009, v. 36, n. 1, pp. 766-777.

http://dx.doi.org/10.1016/j.eswa.2007.10.024

Lin, Chinho; Tseng, Shu-Mei. "Bridging the implementation gaps in the knowledge management system for enhancing corporate performance". Expert systems with applications, 2005 , v. 29, n. 1 , pp. 163-173.

http://dx.doi.org/10.1016/j.eswa.2005.01.015

Lindner, Frank; Wald, Andreas. "Success factors of knowledge management in temporary organizations". Int journal of project management, 2010, v. 29, pp. 877-888.

http://dx.doi.org/10.1016/j.ijproman.2010.09.003 
Macintosh, Ann. Position paper on knowledge assets management. Artificial Intelligence Applications Institute, University of Edinburgh, Scotland, May 1996.

Marwick, Alan. "Knowledge management technology". IBM systems journal, 2001, v. 40, n. 4, pp. 814-830. http://dx.doi.org/10.1147/sj.404.0814

McQueen, Robert. "Four views of knowledge and knowledge management". En: Procs of the $4^{\text {th }}$ Americas conf on information systems, Aug 1998, pp. 609-611.

Nevo, Dorit; Chan, Yolande. "A Delphi study of knowledge management systems". Information \& management, 2007, v. 44 , n. 6 , pp. $583-597$.

http://dx.doi.org/10.1016/j.im.2007.06.001

Newman, Brian; Conrad, Kurt. "A framework for characterizing knowledge management methods, practice, and technologies". En: Procs of the Practical aspects of knowledge management (PAKM 2000), The Knowledge Management Forum, Basel, Switzerland, 2000.

Nonaka, Ikujiro. "A dynamic theory of organizational knowledge creation". Organization science, 1994, v. 5, n. 1, pp. 14-37.

Nonaka, Ikujiro; Takeuchi, Hirotaka. The knowledge-creating company. How Japanese companies create the dynamics of innovations. Oxford University Press, Nueva York, 1995. ISBN: 9780195092691

Olave, Yesid; Gómez, Luis. "IndiSIO: Instrumento de indagación sobre los sistemas de información en las organizaciones". UIS ingenierías, 2002, v. 1, n. 2, pp. 51-70.

Olave, Yesid; Gómez, Luis. "Una reflexión sistémica sobre los fundamentos conceptuales para sistemas de información". Revista colombiana de computación, 2007, v. 8, n. 1, pp. 71-92.

Prahalad, Coimbatore; Hamel, Gary. "The core competence of the corporation". Harvard business review, 1990, v. 68, n. 3, pp. 79-91.

http://faculty.fuqua.duke.edu/ charlesw/s591/willstuff/ oldstuff/PhD_2007-2008/Papers/C08/Prahalad_Hamel_ 1990.pdf

Powell, John; Swart, Juani. "Mapping the values in B2B relationships: a systemic, knowledge-based perspective". Industrial marketing management, 2010, junio, v. 39, n. 3, pp. 437-449.

http://dx.doi.org/10.1016/j.indmarman.2008.11.011

Qureshi, Sajda; Hlupic, Vlatka; Briggs, Robert. “On the convergence of knowledge management and groupware". En: Procs of the $10^{\text {th }}$ intl workshop on groupware (Criwg'2004), Springer, San Carlos, Costa Rica, 2004, pp. 25-33.

Rao, Madanmohan. "Overview: the social life of KM tools". En: Madanmohan, Rao (ed.). Knowledge management tools and techniques, Amsterdam: Elsevier, 2005, pp. 1-73. ISBN: 9780750678186

Richardson, Sandra; Courtney, James; Haynes, John. "Theoretical principles for knowledge management system design: application to pediatric bipolar disorder". Decision support systems, 2006, v. 42, n. 3, pp. 1321-1337. http://dx.doi.org/10.1016/j.dss.2005.11.001

Roberts, Joanne. "Knowledge systems and global advertising services". Creativity and innovation management, 2000, sept., v. 9, n. 3, pp. 163-170.

http://dx.doi.org/10.1111/1467-8691.00169

Rodríguez, Óscar; Martínez, Ana; Vizcaíno, Aurora; Favela, Jesús; Piattini, Mario. "A framework to analyze information systems as knowledge flow facilitators". Information and software technology, 2008, v. 50, n. 6, pp. 481-498. http://dx.doi.org/10.1016/j.infsof.2007.07.002

Rubenstein, Bonnie; Liebowitz, Jay; Buchwalter, Judah; McCaw, Douglas; Newman, Butler; Rebeck, Kenneth. "A systems thinking framework for knowledge management". Decision support systems, 2001, v. 31, n. 1, pp. 5-16. http://dx.doi.org/10.1016/S0167-9236(00)00116-0

Ruggles, Rudy. "The state of the notion: knowledge management in practice". California management review, 1998, v. 40, n. 3, pp. $80-89$.

http://www.nipc.net/km/article/km_group/Ruggles-1998State_of_the_Notion.pdf

Rus, loana; Lindvall, Mikael; Sinha, Sachin. "Knowledge management in software engineering: a state of the art report". Data \& analysis center for software, 2001, 53 pp.

Sánchez, Ron. "Managing knowledge into competence: the five learning cycles of the competent organization". En: Sánchez, Ron (ed.). Knowledge management and organizational competence. Nueva York: Oxford University Press, 2001, pp. 3-37. ISBN: 9780199259281

http://dx.doi.org/10.1093/acprof:oso/9780199259281.003. 0001

Sher, Peter; Lee, Vivid. "Information technology as a facilitator for enhancing dynamic capabilities through knowledge management". Information \& management, 2004, v. 41, n. 8, pp. 933-945.

http://dx.doi.org/10.1016/j.im.2003.06.004

Schindler, Martin; Eppler, Martin. "Harvesting project knowledge: a review of project learning methods and success factors". Intl journal of project management, 2003, v. 21, n. 3, pp. 219-228.

http://www.uncg.edu/bae/people/amoako/ISM654/rea ding_\%2323.pdf

Skok, Walter; Kalmanovitch, Caroline. "Evaluating the role and effectiveness of an intranet in facilitating knowledge management: a case study at Surrey County Council". Information \& management, 2005, v. 42, pp. 731-744.

http://www2.warwick.ac.uk/fac/soc/wbs/conf/olkc/archi ve/oklc3/papers/id261.pdf

Spender, John. "Making knowledge the basis of a dynamic theory of the firm". Strategic management journal, 1996, v. 17, (special issue: Knowledge and the firm), pp. 45-62. http://www.jcspender.com/uploads/Spender-SMJ96SI.pdf

Spiegler, Israel. "Technology and knowledge: bridging a 'generating' gap". Information \& management, 2003, v. 40, n. 6, pp. 533-539.

http://dx.doi.org/10.1016/S0378-7206(02)00069-1 
Stalk, Georg; Evans, Philip; Shulman, Lawrence. "Competing on capabilities: the new rules of corporate strategy". Harvard business review, 1992, v. 70, n. 2, pp. 57-69.

Teece, David; Pisano, Gary; Shuen, Amy. "Dynamic capabilities and strategic management". Strategic management journal, 1997, v. 18, n. 7, pp. 509-533.

http://faculty.fuqua.duke.edu/ charlesw/s591/BocconiDuke/Papers/C10/TeecePisanoShuenSMJ.pdf

Tiwana, Amrit; Ramesh, Balasubramaniam. "Integrating knowledge on the web". IEEE internet computing, 2001, v. 5, pp. 32-39.

http://www.inf.ufes.br/ falbo/download/aulas/teng soft/2007-2/Texto8.pdf

Tseng, Shu-Mei. "The effects of information technology on knowledge management systems". Expert systems with applications, 2008 , v. 35 , pp. 150-160.

http://dx.doi.org/10.1016/j.eswa.2007.06.011

Valente, Andre; Housel, Thomas. "A framework to analyze and compare knowledge management tools". En: Procs of the Knowledge-based intelligent information engineering and allied technologies (KES'2002), Crema, Italy, 2002, pp. 291-295.

Van-Elst, Ludger; Dignum, Virginia; Abecker, Andreas. "Towards agent-mediated knowledge management". En: Procs of the int symposium AMKM 2003, Stanford, CA, USA, 2003, pp. 1-30. ISBN: 9783540208686

http://www.springerlink.com/content/7w83u24a12wv5 fco/fulltext.pdf

Vizcaíno, Aurora; Piattini, Mario; Martínez, Manuel; Aranda, Gabriela. "Evaluating collaborative applications from a knowledge management approach". En: Procs of the Evaluation of collaborative information systems and support for virtual enterprises (ECE), Linkoping, Sweden, 2005. ISBN: 0 769523625
http://doi.ieeecomputersociety.org/10.1109/WETICE. 2005.36

Von-Krogh, Georg. "Care in knowledge creation". California management review, 1998, v. 40, n. 3, pp. 133-153.

Wiig, Karl. "Integrating intellectual capital and knowledge management". Long rang planning, 1997, v. 30, n. 3, pp. 399-405.

http://dx.doi.org/10.1016/S0024-6301(97)90256-9

Woitsch, Robert; Karagiannis, Dimitris. "Process-oriented knowledge management systems based on KM-services: the Promote approach". Intl journal of intelligent systems in accounting, finance \& management, 2002, v. 11, n. 4, pp. 253-267.

http://dx.doi.org/10.1002/isaf.235

Yim, Nam-Hong; Kim, Soung-Hie; Kim, Hee-Woong; Kwahk, Kee-Young. "Knowledge based decision making on higher level strategic concerns: system dynamics approach". Expert systems with applications, 2004, v. 27, n. 1, pp. 143-158. http://dx.doi.org/10.1016/j.eswa.2003.12.019

Zack, Michael. "An architecture for managing explicated knowledge". Sloan management review, 1998, Sept, v. 39, n. 4, pp. 45-58.

Zhao, Jingyuan. "School knowledge management framework and strategies: the new perspective on teacher professional development". Computers in human behavior, 2010, v. 26 , n. 2, pp. $168-175$.

http://dx.doi.org/10.1016/j.chb.2009.10.009

Zollo, Maurizio; Winter, Sidney. "Deliberate learning and the evolution of dynamic capabilities". Organization science, 2002, v. 13, n. 3, pp. 339-351.

http://dx.doi.org/10.1287/orsc.13.3.339.2780

\section{Si te interesan los}

\section{NDICADORES EN IENGIA E ENOLOGÍA,}

y todos los temas relacionados cony the melieion de la ciencia, tales como:

Análisis de citas, Normaukater. la ciencia en la sociedac. Inom, ios So elo logia de la ciencia, Política científica, Comunicación de Ja ciene M Revistas, Bases de datos, Índices de impacto, Políticas de open acces se Análisis de la nueva economía, Mujer y ciencia, etc:

\section{Entonces INCYT es tu lista. Suscribete en:}

\section{http://www.rediris.es/list/info/incyt.htmI}

